\title{
U - CITY VÀ MÔ HÌNH TẠI HÀN QUỐC
}

\author{
CN. TRẦN TH! HÔNG HÀ, TS. PHẠM MINH HẢl \\ Viện Khoa học Đo đạc và Bản đồ
}

\section{U- city - "Thành phố thông minh"}

Hiện nay, khái niệm "U-city” trở nên phổ biến trên thế giới với mục đích xây dựng những thành phố thông minh, có nhiều dịch vụ sử dụng dữ liệu thông tin địa lý để nâng cao chất lượng môi trường sống của con người.

"U-City" còn được gọi là thành phố thông minh. Thành phố thông minh là nơi hệ thống thông tin được chia sẻ sử dụng công nghệ điện toán đám mây. "U-City" ("U" là viết tắt của từ tiếng Anh "Ubiquitous" có nghĩa là “mọi nơi”) là một khái niệm mới, ra đời lần đầu tiên ở Hàn Quốc năm 1998 và đang dần thành xu hướng mới của trong quy hoạch đô thị trên thế giới. U-city được xây dựng dựa trên nền tảng của sự phát triển của công nghệ thông tin và ngành công nghiệp dư liệu địa lý. Chi tiết hơn, việc xây dựng Ucity dựa trên 3 công nghệ chính đó là ITS, GIS, và Green Home. Nó tạo điều kiện cho cư dân đô thị có điều kiện sống thuận lợi bằng các cơ sở hạ tầng hiện đại và dịch vụ số được kết nối mọi lúc mọi nơi. (Xem hình 1)

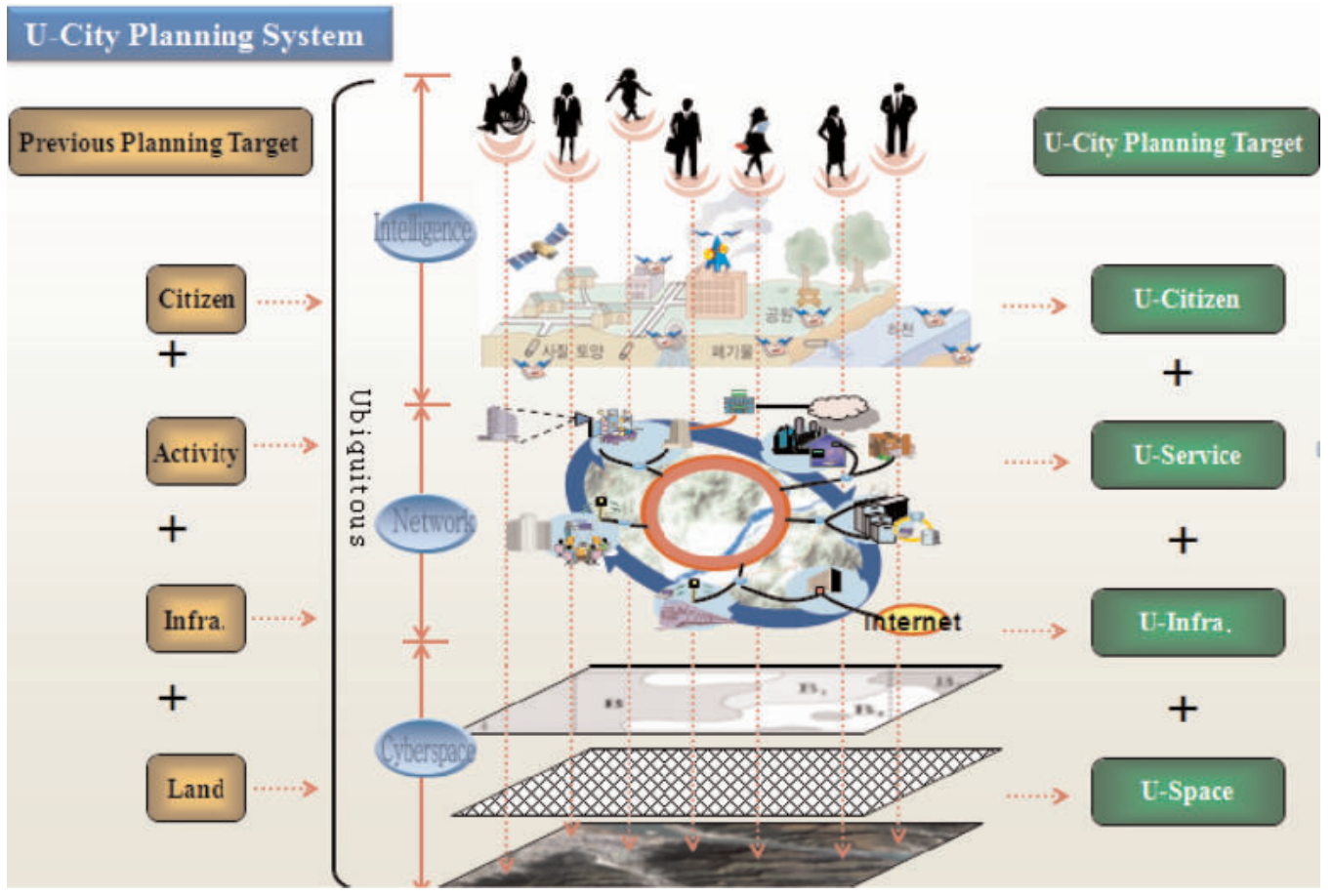

Hình 1: Khái niệm U-city 
Không gian đô thị trong U-city đề cập đến các không gian sinh sống và làm việc của công dân đô thị như khu làm việc, nhà ở, công viên. Sự ứng dụng công nghệ thông tin khắp mọi nơi trong thành phố bao gồm các hạ tầng như hệ thống các trang thiết bị công nghệ thông tin như mạng, trung tâm điều hành... cung cấp dịch vụ cho nhu cầu thực tế của người sử dụng.

U-city cung cấp đầy đủ các thông tin liên quan đến nơ sinh hoạt của cư dân đô thị. Một cư dân đô thị có thể không cần ở nhà nhưng vẫn biết tình trạng trong nhà, trong căn hộ của mình, và có thể điều khiển bật/tắt các thiết bị qua các thiết bị kết nối mạng Internet không dây. Bên cạnh đó, cũng có thể theo dõi con cái và liên lạc với người thân trong gia đình thông qua hệ thống video surveillance qua mạng, hoặc quản lý người vào/ra khỏi nhà của mình.

Do vậy, U-city mang lại những giá trị lớn khi làm cho năng lượng được sử dụng hiệu quả hơn và các hoạt động liên quan đến cuộc sống của con người được đồng bộ hóa một cách dễ dàng hơn.

Sau hơn 25 năm nghiên cứu ứng dụng các giải pháp tiên tiến vào việc xây dựng hạ tầng công nghệ thông tin và quy hoạch đô thị, cùng với bộ luật chính sách xây dựng tổng thể về Quốc gia thông minh "U-Korea", Hàn Quốc đã trở thành quốc gia đi đầu trong việc triển khai các đô thị thông minh với 15 dự án lớn và thành công mang tầm quốc tế của thành phố Songdo, Incheon. Các mô hình và giải pháp của quốc gia Hàn Quốc đã được chia sẻ thông qua các diễn đàn hợp tác tại hơn 20 quốc gia như Colombia, UAE...[1]. (Xem hình 2)

\section{Mô hình triển khai U-city tại Hàn Quốc}

2.1. Bối cảnh ra đời U- city tại Hàn Quốc

Nếu cách đây 30 năm tổng sản phẩm quốc nội của Hàn Quốc chỉ đứng ngang với các nước nghèo ở châu Phi và châu Á thì hiện nay, tổng sản phẩm quốc nội của Hàn Quốc xếp thứ 10 trên thế giới. Với sự cải cách kịp thời và phù hợp, Hàn Quốc hiện là một trong những quốc gia phát triển nhất trên thế giới, đặc biệt trong lĩnh vực công nghệ thông tin và truyền thông với nhiều công ty lớn của Hàn Quốc bắt đầu tạo chỗ đứng trên thị trường thế giới.

Tuy nhiên, trong những thập niên gần

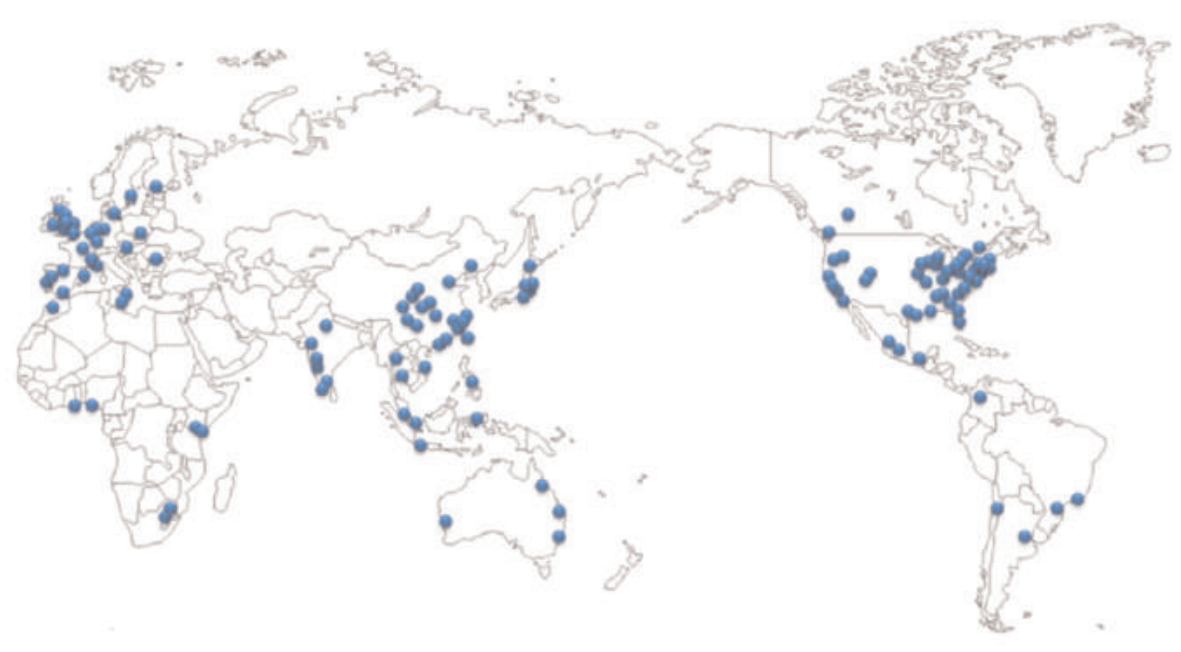

Hình 2: Các dụ̣ án thành phố thông minh trên thế giới (2012) [1] 
đây, sự phát triển mạnh mẽ về kinh tế - xã hội, quá trình đô thị hóa nhanh chóng cùng với sự bùng nổ dân số đã tạo những áp lực lớn lên môi trường sống. Đồng thời, sự cạnh tranh mạnh mẽ trong lĩnh vực công nghệ thông tin giữa các công ty cũng như giữa các quốc gia, nhu cầu đảm bảo cuộc sống phát triển và bền vững cho cộng đồng đã đặt ra bài toán cho các nhà quản lý và hoạch định chính sách về việc xây dựng và phát triển một thành phố thông minh, nơ mà con người có thể sử dụng công nghệ để phục vụ cuộc sống, gắn kết cộng đồng

\subsection{Kết quả mong đợi từ U-city}

Việc phát triển và mở rộng các thành phố thông minh được trông đợi tạo điều kiện nâng cao hiệu quả quản lý đô thị thông qua việc giải quyết tình trạng ùn tắc giao thông, giảm chi phí hậu cần và nâng cao hiệu quả quản lý thiết bị, cung cấp các "dịch vụ thông minh" đa dạng liên quan đến giao thông, an ninh, giáo dục và sức khỏe, nâng cao chất lượng cuộc sống.

* Đối với chính quyền địa phương

Nâng cao hiệu quả quản lý lãnh thổ

Phát triển ngành công nghiệp mới trong tương lai

Tạo ra nhiều việc làm
Quản lý đô thị hiệu quả

Tạo các giá trị lớn cho thành phố

Chuẩn bị cơ sở cho phát triển địa phương

* Đối với công ty tư nhân:

Tạo điều kiện tìm kiếm thị trường mới

Mở rộng cơ hội tham gia vào các dự án

Tăng lợi nhuận nhờ tiết giảm năng lượng sử dụng

* Đối với người dân:

Cung cấp các dịch vụ đô thị

Đảm bảo cuộc sống thuận lợi, an toàn.

\section{Chiến lược phát triển U-city của Hàn Quốc}

3.1. Quy hoạch phát triển thành phố thông minh

Quy định về xây dựng thành phố thông minh được chính phủ Hàn Quốc đưa ra và có hiệu lực từ ngày 29 tháng 9 năm 2008, bao gồm các điều lệ về lập kế hoạch, xây dựng và quản lý có hiệu quả thành phố thông minh [1]. (Xem bảng 1)

3.2. Kế hoạch phát triển toàn diện thành phố thông minh của Hàn Quốc

3.2.1. Kế hoạch 5 năm lần thứ nhất

Bảng 1: Phương pháp tiếp cận xây dựng thành phố thông minh

\begin{tabular}{|l|l|}
\hline \multicolumn{1}{|c|}{ Giới thiệu } & \multicolumn{1}{c|}{ Định nghĩa, thành phần, tiêu chuẩn về dịch vụ và công nghệ } \\
\hline Lập kế hoạch & Thủ tục của kế hoạch phát triển toàn diện thành phố thông minh \\
\hline Xây dựng & $\begin{array}{l}\text { - Thành lập Ủy ban Thành phố thông minh (Trung ương) và Hội đồng (chính quyền } \\
\text { - Tiến hành xây dựng thủ tục của thành phố thông minh }\end{array}$ \\
\hline Hoạt động & $\begin{array}{l}\text { Quy chế quản lý/vận hành, hỗ trợ và tài trợ của Chính phủ/chính quyền địa } \\
\text { phương phấ triên nguồn nhân lực, quản lý thông tin thành phố thông minh }\end{array}$ \\
\hline
\end{tabular}


(2009 - 2013). (Xem bảng 2)

3.2.2. Chiến lược chính thúc đẩy phát triển thành phố thông minh

Bộ Đất đai, Giao thông và Hàng hải Hàn Quốc đã soạn thảo 4 chiến lược chính cho khung thể chế để xây dựng U-city và xúc tiến toàn diện xây dựng U-City như $R \& D$ (research \& development - nghiên cứu và phát triển), hỗ trợ các ngành công nghiệp liên quan và đầu tư hỗ trợ các chuyên gia đào tạo. (Xem bảng 3)

\section{Phát triển thành phố thông minh tại Việt Nam}

Mô hình thành phố thông minh đã được tập đoàn IBM triển khai trên toàn thế giới. Hơn một nửa số dự án này là tại khu vực

Bảng 2: Kế hoạch 5 năm lần thứ nhất phát triển U-city

\begin{tabular}{|c|c|c|c|}
\hline Tầm nhìn & \multicolumn{3}{|c|}{$\begin{array}{l}\text { Xây dựng một thành phố thông minh hiện đại, tăng cường chất lượng } \\
\text { cuộc sống và phát triển toàn diện thành phố }\end{array}$} \\
\hline Mục tiêu & $\begin{array}{l}\text { Hiệu quả quản lý } \\
\text { đô thị }\end{array}$ & $\begin{array}{l}\text { Thúc đẩy sự phát triển của nền } \\
\text { công nghiệp năng lượng mới }\end{array}$ & \begin{tabular}{|c|} 
Dịch vụ đô thị cao \\
cấp
\end{tabular} \\
\hline \multirow{4}{*}{$\begin{array}{c}\text { Đẩy mạnh } \\
\text { chiến lược và } \\
\text { thực hiện nhiệm vụ }\end{array}$} & $\begin{array}{l}\text { Sự chuẩn bị của } \\
\text { các tổ chức }\end{array}$ & \multicolumn{2}{|c|}{$\begin{array}{l}\text { - Chuẩn bị các định hướng và hướng dẫn cho việc xây } \\
\text { dựng và quản lý thành phố thông minh } \\
\text { - Bảo vệ các thông tin cá nhân } \\
\text { - Phòng chống thiên tai, tai nạn và thiệt hại } \\
\text { - Thành lập các tiêu chuẩn công nghệ thành phố thông } \\
\text { minh } \\
\text { - Chuẩn bị các phương pháp để phân phối thông tin } \\
\text { thành phố thông minh }\end{array}$} \\
\hline & $\begin{array}{l}\text { Phát triển công } \\
\text { nghệ cốt lõi }\end{array}$ & \multicolumn{2}{|c|}{$\begin{array}{l}\text { - Phát triển nhanh chóng các công nghê̂ cốt lõi tiên tiến } \\
\text { cho các thành phần chính của thành phố thông minh } \\
\text { - Phát triển công nghệ để thu thập, xử lý và sử dụng } \\
\text { thông tin }\end{array}$} \\
\hline & $\begin{array}{l}\text { Hỗ trợ tăng trưởng } \\
\text { công nghiệp }\end{array}$ & \multicolumn{2}{|c|}{$\begin{array}{l}\text { - Tào ra mô hình thành công bằng cách hỗ trợ mô hình } \\
\text { thành phố thông minh } \\
\text { - Phát triển nguồn tài nguyên nhân lực cho nền công } \\
\text { nghiệp thành phố thông minh }\end{array}$} \\
\hline & $\begin{array}{l}\text { Tạo ra dịch vụ } \\
\text { hợp lý }\end{array}$ & \multicolumn{2}{|c|}{$\begin{array}{l}\text { Cung cấp các dịch vụ khác nhau mà mọi người có thể } \\
\text { trải nghiệm, dựa trên thành phố thông minh: } \\
\text { - Thành lập hệ thống hành chính thông minh } \\
\text { - Cung cấp dịch vụ vận chuyển phù hợp } \\
\text { - Xây dựng các kế hoạch phát triển dịch vụ tiên tiến } \\
\text { - Cung cấp dịch vụ xanh thân thiện với môi trường } \\
\text { - Xây dựng hệ thống phản ứng và phòng vệ thông } \\
\text { minh }\end{array}$} \\
\hline
\end{tabular}


châu Á. Tại Việt Nam, theo đại diện IBM, với thành công bước đầu tại Đà Nãng, mô hình thành phố thông minh được kỳ vọng tiếp tục phát triển tại Huế và Khánh Hoà.[2]

Hình 3: Ứng dụng CNTT xây dựng thành

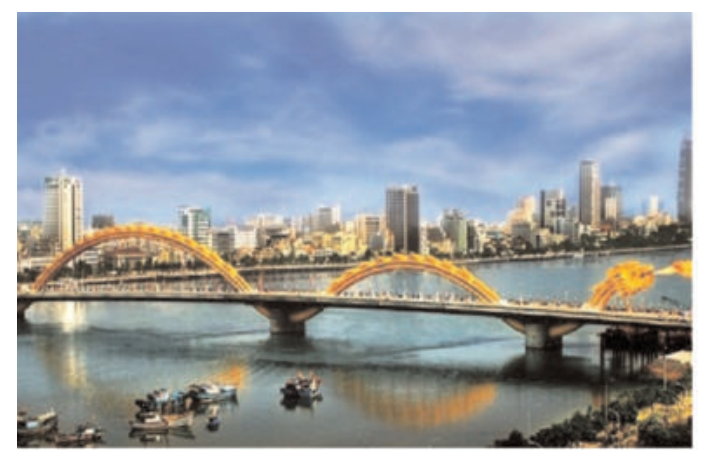

phố thông minh tại Đà Nã̃ng

(Ảnh: Chinhphu.vn)

Từ tháng 5/2012, Đà Nã̃ng là địa phương đầu tiên của Việt Nam và là một trong 33 thành phố trên toàn thế giới được tập đoàn IBM hỗ trợ phát triển dự án "Thành phố thông minh hơn". Sau hơn 1 năm triển khai, dự án xây dựng "Thành phố thông minh hơn" đã cho thấy những hiệu quả của việc áp dụng khoa học công nghệ tiên tiến vào công tác quản lý tài nguyên và hệ thống hạ tầng, nâng cao chất lượng cuộc sống người dân. Điều này đã mở ra triển vọng nhân rộng mô hình thành phố thông minh ra các địa phương khác của Việt Nam. Những thiết bị cảm biến tự động đo lường độ đục, độ mặn, độ dẫn điện, độ $\mathrm{pH}$ và nồng độ clo trong nước... đã giúp công ty cấp nước Đà Nẵng đảm bảo nguồn nước sạch cho người dân. Trung tâm điều hành giao thông thông minh tự động cung cấp thông tin lịch trình, vị trí, tốc độ... của 100 xe bus và cập nhật lên cổng thông tin điện tử của Sở giao thông vận tải để hành khách tham khảo. Tuy nhiên, áp dụng hệ thống công nghệ cao

Bảng 3: Các chiến lược chính thúc đẩy phát triển thành phố thông minh

\begin{tabular}{|c|c|c|c|c|}
\hline $\begin{array}{l}\text { Chiến lược } \\
\text { chính }\end{array}$ & $\begin{array}{l}\text { Nền tảng của } \\
\text { hệ thống pháp luật }\end{array}$ & $\begin{array}{c}\text { Phát triển công } \\
\text { nghệ cốt lõi }\end{array}$ & $\begin{array}{c}\text { Hỗ trợ } \\
\text { công nghiệp }\end{array}$ & $\begin{array}{l}\text { Đào tạo các } \\
\text { chuyên gia }\end{array}$ \\
\hline \multirow[b]{2}{*}{ Chi tiết } & $\begin{array}{l}\text { Hỗ trợ kế hoạch, xây } \\
\text { dựng và quản lý }\end{array}$ & $\begin{array}{l}\text { Phát triển công } \\
\text { nghệ cốt lõi như } \\
\text { tích hợp U-city, tiêu } \\
\text { chuẩn dịch vụ của } \\
\text { U-city }\end{array}$ & $\begin{array}{l}\text { Hỗ trợ kế hoạch } \\
\text { phát triển và tài trợ } \\
\text { cho các ngành } \\
\text { công nghiệp liên } \\
\text { quan tới U-city }\end{array}$ & $\begin{array}{l}\text { Đào tạo các } \\
\text { chuyên gia cho lĩnh } \\
\text { vực U-city }\end{array}$ \\
\hline & $\begin{array}{l}\text { Thành lập: } \\
\text { - Kế hoạch phát triển } \\
\text { toàn diện U-city } \\
\text { - Chính sách xây dựng } \\
\text { U-city } \\
\text { - Chính sách công nghệ } \\
\text { tích hợp xây dựng với } \\
\text { CNTT } \\
\text { - Chính sách quản lý U- } \\
\text { city } \\
\text { - Tiêu chuẩn dịch vụ U- } \\
\text { city } \\
\text { - Tiêu chuẩn công nghệ } \\
\text { kỹ thuật U-city }\end{array}$ & $\begin{array}{l}\text { - Nghiên cứu và } \\
\text { phát triển U-Eco } \\
\text { City (thành phố } \\
\text { xanh thông minh) } \\
\text { - Phát triển công } \\
\text { nghệ U-city trong } \\
\text { nước, phục vụ cho } \\
\text { xuất khẩu } \\
\text { - Xây dựng mô } \\
\text { hình chuẩn U-city } \\
\text { cho các thị trấn mới }\end{array}$ & $\begin{array}{l}\text { - Xây dựng các khu } \\
\text { vực hành chính } \\
\text { đặc biệt cho thử } \\
\text { nghiệm U-city } \\
\text { - Thúc đẩy sự phát } \\
\text { triển của các ngành } \\
\text { công nghiệp liên } \\
\text { quan tới U-city }\end{array}$ & $\begin{array}{l}\text { - Đào tạo nguồn } \\
\text { nhân lực chất } \\
\text { lượng cao } \\
\text { - Đào tạo chuyên } \\
\text { gia có chuyên môn } \\
\text { kỹ thuật } \\
\text { - Đào tạo cán bộ }\end{array}$ \\
\hline
\end{tabular}


để đưa vào phát triển đô thị vẫn còn ẩn chứa nhiều thách thức đối với Việt Nam. Thách thức lớn nhất hiện nay là điều kiện phát triển cho các đô thị và công trình của Việt Nam chưa đồng bộ, và hạ tầng cũng chưa được phát triển toàn diện. Cho nên khi lồng ghép hệ thống công nghệ cao vào thì sẽ có những khó khăn nhất định. Vấn đề thứ hai là sự tiếp nhận của người dân, đặc biệt là người quản lý đô thị. Để sở hữu những căn hộ thông minh trong khu đô thị thông minh thì người dân bắt buộc phải chi trả một chi phí nhất định trong quá trình sử dụng, và chắc chắn là không rẻ. Vì vậy, điều quan trọng là cần phải nâng cao nhận thức cho người dân để nhận thấy sự khác biệt giữa khu đô thị cũ và đô thị thông minh, đồng thời, các cấp và ban ngành có liên quan cần đưa ra những quy chuẩn thích hợp, và cả những nghị định mới nhằm quản lý các dự án đô thị, trách nhiệm giữa cái cũ và mới để quản lý đồng bộ và có những chính sách thu hút các nhà đầu tư để phát triển thí điểm các công trình thông minh nhằm giúp nâng cao chất lượng cuộc sống cho từng hộ gia đình.

\section{Kết luận}

Dự án U-city - Thành phố thông minh đã được tiến hành và triển khai ở nhiều quốc gia trên thế giới với mục đích xây dựng những thành phố thông minh, có nhiều dịch vụ sử dụng dữ liệu thông tin địa lý để nâng cao chất lượng môi trường sống của con người. Hàn Quốc là một trong những quốc gia tiên phong ở châu Á trong việc xây dựng và phát triển thành phố thông minh. (Hàn Quốc đã tiến hành xây dựng 50 thành phố thông minh (trên tổng số 163 thành phố hiện nay - chiếm 30,67\%) [1]. Tuy nhiên, hiện nay, các dự án quy hoạch và phát triển thành phố thông minh ở Hàn Quốc đang bị thu hẹp và trì hoãn do sự thiếu chuyên nghiệp và thiếu hệ thống tổ chức và còn gặp nhiều trở ngại về tài chính do sự suy thoái kinh tế. Hơn nữa, sự phát triển mới tập trung ở khu vực đô thị mới và dịch vụ công cộng và tập trung chủ yếu vào thị trường nội địa.

Tại Việt Nam, việc ứng dụng công nghệ thông tin để tăng cường khả năng quản lý tài nguyên và hệ thống hạ tầng là bước đi mang tầm chiến lược cần được quan tâm đầu tư để nâng cao chất lượng cuộc sống người dân. Với những thành công bước đầu trong việc xây dựng thành phố thông minh ở Đà Nẵng và sắp tới được kỳ vọng ở Huế, Việt Nam đang từng bước hội nhập với thế giới trong lĩnh vực phát triển khoa học công nghệ phục vụ đời sống. Tuy nhiên, ở những khu vực khác nhau trên thế giới sẽ có những nhu cầu và hạn chế khác nhau, cơ sở hạ tầng điện, viễn thông khác nhau, điều kiện khí hậu và quan điểm chính trị khác nhau. Để có thể hướng tới một sự phát triển bền vững và toàn diện, các nhà quản lý và hoạch định chính sách cần có những giải pháp chuyển đổi mới, thích ứng với sự thay đổi của môi trường kinh tế - xã hội nhanh chóng hiện nay cũng như đáp ứng được nhu cầu của người dân. Các giải pháp có thể bao gồm: (i) Thiết kế, xây dựng và thành lập các tiêu chuẩn, thủ tục liên quan tới thành phố thông minh; (ii) Bên cạnh khu đô thị mới thì việc quan tâm và xây dựng thành phố thông minh ở khu vực phố cổ là cần thiết; (iii) Phát triển mô hình thành phố "thông minh và xanh"; đẩy mạnh quan hệ hợp tác quốc tế; (iv) Cung cấp các dịch vụ quản lý đô thị hợp lý, thuận lợi cho người dân địa phương. $O$

\section{Tài liệu tham khảo}

[1]. "Curent status and prospect of U-city Policy in Korea", Hội thảo và diễn đàn doanh nghiệp Việt Nam - Hàn Quốc về xây dựng thành phố thông minh và hạ tầng dữ liệu không gian Quốc gia (Korea spatial data \& U - city vision in Vietnam), Hà Nội, 24-42013.

[2]. Trần Long, Phát triển thành phố thông minh tại Việt Nam. Nguồn: 
http://vtv.vn/san-pham/phat-trien-thanhpho-thong-minh-tai-viet-nam/78432.vtv.

[3]. Phạm Bùi. Việt Nam bắt đầu xây dựng "đô thị thông minh. Nguồn:

h $t \mathrm{t} p: / / w$ w $w$. $\mathrm{p} p \mathrm{t}-$ hanoi.com.vn/portal/SmartPhone/SmartPag es/tintuc chitiet.aspx?new id $=3386$
[4]. Phan Châu. Những thành phố thông minh hơn. Nguồn:

http://www.pcworld.com.vn/articles/tieud $\mathrm{u} n \mathrm{~g} / \mathrm{s}$ o $\mathrm{ng}$ - c o n g nghe/2014/03/1234600/nhung-thanh-phothong-minh-hon/O

\section{Summary}

\section{Ubiquitous City (U - City) and Korean Ubiquitous City Model}

BSc. Tran Thi Hong Ha, Dr. Pham Minh Hai

\section{Institute of Geodesy and Cartography}

Currently, the concept of Ubiquitous-City ( $U$ - City) is becoming more popular with the purpose of building smart cities where have many data services using geographic information to improve the quality of life. "U - City" first launched in Korea in 1998 is gradually becoming a new trend in urban planning in the world. This article introduces the general overview of $U$ - City and $U$ - City development model in South Korea as well as the assessment made on the application and development of $U$ - city in Vietnam. $O$

Ngày nhận bài: 13/5/2014.

\section{ỨNG DỤNG CÔNG NGHỆ GIS.....}

(Tiếp theo trang 51)

[11]. UNEP (1997). National land degradation assessment and mapping in Kenia.
[12]. UN (1992). Convention to Combat Desertification (UNCCD). $\bigcirc$

\section{Summary}

\section{Application of GIS technology in mapping potential land degradation of Tay Nguyen region}

Dr. Luu The Anh, MSc. Nguyen Ngoc Thanh, Eng. Hoang Quoc Nam

Institute of Geography - Vietnam Academy of Science and Technology

Potential land degradation map is a one of thematic maps in geographical mapping system. It plays an important role in the management and sustainable use of land resources. This paper presents a summary of the steps and processes of GIS-based potential land degradation mapping in the Central Highlands region at scale of 1:250.000 according to the integrated geographical approach. The result map shows four severe classes of land degradation potential in the Central Highlands, in which area of the low potential class (TN1) has $1.528 .933,0$ ha (accounted for $28 \%$ of total area), moderated potential class (TN2) has 2.059.142,8 ha (accounted for 37,7\%), high potential class (TN3) covers 1.159.188,9 ha (accounted for 21,2\%); and very high potential class (TN4) has 716.707,4 ha (accounted for $13,1 \%$ ). These findings are the basic information of degradation potential needed to establish land use planning of the Central Highland and proposal of measurements for rational use of land resources. $O$

Ngày nhận bài: 23/5/2014. 